OPEN ACCESS

Edited by:

Michael Graner,

University of Colorado Denver,

United States

Reviewed by:

Anna Bersano,

Fondazione IRCCS Istituto Neurologio

Carlo Besta, Italy

Simona Lattanzi,

Marche Polytechnic University, Italy

Candice Delcourt,

University of New South

Wales, Australia

*Correspondence:

$\mathrm{Xin} \mathrm{Hu}$

huxingxxy@gmail.com

†These authors have contributed equally to this work

Specialty section:

This article was submitted to

Stroke,

a section of the journal

Frontiers in Neurology

Received: 12 November 2020 Accepted: 30 March 2021

Published: 25 May 2021

Citation:

Li Y, Wen D, Cui W, Chen Y, Zhang F, Yuan M, Xiao H, Li H, Ma L, Hu X and You C (2021) The Prognostic Value of the Acute Phase Systemic Immune-Inflammation Index in

Patients With Intracerebral Hemorrhage.

Front. Neurol. 12:628557.

doi: 10.3389/fneur.2021.628557

\section{The Prognostic Value of the Acute Phase Systemic Immune-Inflammation Index in Patients With Intracerebral Hemorrhage}

\author{
Yunke $\mathrm{Li}^{1+}$, Dingke Wen ${ }^{1+}$, Wenyao Cui ${ }^{1+}$, Yuqi Chen ${ }^{1}$, Fazhen Zhang ${ }^{2}$, Maolin Yuan ${ }^{2}$, \\ Han $\mathrm{XiaO}^{2}$, Hao $\mathrm{Li}^{1}$, $\mathrm{Lu} \mathrm{Ma}^{1}$, Xin $\mathrm{Hu}^{1 *}$ and Chao You ${ }^{1}$ \\ ${ }^{1}$ Neurosurgery Department of West China Hospital, Sichuan University, Chengdu, China, ${ }^{2}$ Medical School of Sichuan \\ University, Chengdu, China
}

Background and Purpose: The systemic immune-inflammation index (SII) is a novel prognostic index in various diseases. We evaluated the predictive value of SII in patients with intracerebral hemorrhage $(\mathrm{ICH})$.

Methods: Patients with primary spontaneous $\mathrm{ICH}$ were enrolled. SII was constructed based on peripheral platelet $(P)$, neutrophil $(N)$, and lymphocyte $(L)$ and defined as $P^{*} N / L$. In addition to admission testing, acute phase SII was collected to analyze the potential dynamic change. Poor outcome was defined as modified Rankin Scale of more than 3 at 90 days.

Results: We included 291 patients; 98 (34\%) achieved favorable functional outcomes. Day-1 SII was higher and was more related to poor outcome than was admission SII. Median time of day-1 SIl was $29 \mathrm{~h}$ from onset. Day-1 SIl had an OR in outcome (mRS >3) $1.74(95 \% \mathrm{Cl}=1.03-3.00, p=0.04)$. The binary cutoff point of SII calculated using the area under the curve (AUC) method was 1,700 × 10\% / L, AUC 0.699 $(95 \% \mathrm{Cl}=0.627-0.774)$ (sensitivity $53.3 \%$, specificity $77.3 \%)(\mathrm{OR}=2.36,95 \% \mathrm{Cl}=$ 1.09-5.26, $p=0.03$ ).

Conclusions: SII, especially day-1 SII, was highly associated with 90-day functional outcome in patients with $\mathrm{ICH}$ and could be used to predict outcomes.

Keywords: stroke, prognosis, blood platelets, intracerebral hemorhage, neutrophils lymphocyte ratio

\section{INTRODUCTION}

Spontaneous intracerebral hemorrhage (ICH) is associated with high mortality and poor outcome (1). Studies found various indicators for predicting outcome following ICH (2-4); however, few of these involve biochemical tests. The brain injury after ICH includes the primary injury, which is the mechanical damage of the adjacent tissues by hematoma within the first hours after ICH, and the secondary injury, which is initiated by the extravasation of blood products into the brain parenchyma (5). Mounting preclinical evidence has shown that inflammation after ICH plays an important role in the secondary brain injury (6). Furthermore, clinical 
laboratory results that reflect inflammation have been reported to predict ICH outcome. Platelet (PLT) counts have been associated with growth edema (7), which predicts ICH outcome (8). The neutrophil-lymphocyte ratio (NLR) was reported $(9,10)$, as were PLT-lymphocyte ratio (PLR) (11), and lymphocytemonocyte ratio (LMR) (12). These studies showed that levels of inflammation are highly related to the clinical outcome following ICH (13).

The systemic immune-inflammation index (SII), which was calculated as peripheral platelet*neutrophil/lymphocyte, was first reported as a prediction tool in cancers such as hepatocellular carcinoma (14-16). In 5,602 coronary artery disease patients after coronary intervention, SII was shown to have a better prediction for major cardiovascular events than traditional risk factor (17). In acute ischemic stroke, SII was reported as an independent risk factor for stroke severity (18). Moreover, dynamic changes of SII was suggested as a promising prognostic predictor for cancer patients such as colorectal cancer and hepatocellular carcinoma $(19,20)$. A recent study confirmed the value of SII for predicting short-term outcome following ICH (21). Nevertheless, the role of SII in predicting long-term outcome following $\mathrm{ICH}$ is unknown.

In the present study, we investigated acute phase SII and favorable outcome of ICH in recovery. We also studied dynamic changes of SII to identify a more precise way to predict outcome.

\section{METHODS}

The data and code that support the findings of this study are available from the corresponding author upon reasonable request. The study was approved by the local ethic committee.

We retrospectively collected data from patients admitted to West China Hospital, Sichuan University (Sichuan, China) from February 2018 to February 2019. The inclusion criteria were as follows: (1) over 18 years of age; (2) admission diagnosis of ICH based on brain CT scans; (3) $<24 \mathrm{~h}$ from onset to admission; (4) available clinical data including at least one laboratory test of platelets, neutrophils, lymphocytes and monocytes; and (5) neuro-image to evaluate the characteristics of the hematoma. Exclusion criteria were as follows: (1) secondary ICH (aneurysm, vascular malformation, or tumor); (2) possible disease that may affect laboratory results (leukemia, lymphoma, or thrombocytopenia); and (3) unavailability of outcome data; (4) patients with coagulopathy or anticoagulant therapy; and (5) patients with active infection or autoimmune disease.

We recorded age, sex, clinical record, previous medical history, laboratory results (PLT), absolute neutrophil count (ANC), absolute lymphocyte count (ALC), absolute monocyte count (AMC), baseline CT imaging characteristics, and surgical information if available. ICH volume was measured based on the $\mathrm{ABC} / 2$ method (22). We collected all laboratory test results during hospitalization. SII was defined as platelet*neutrophil/lymphocyte.

The primary outcome was modified Rankin scale (mRS) at 90 days from onset. mRS was measured at outpatient visit or by telephone using a structured interview (23). We defined favorable outcome as mRS 0-3, and unfavorable outcome was mRS 4-6.

All statistical analyses were performed using $\mathrm{R}$ software (Version 4.0.2, R Core Team, Vienna, Austria). Continuous variables were tested using the Student's $t$ test or Mann-Whitney test and were expressed as mean (standard deviation) or median (interquartile range) depending on their distribution. Categorical variables were defined as numbers and were analyzed using the $\chi^{2}$ test or Fisher exact test. Receiver operating curves (ROCs) were generated and the area under curve (AUC) was calculated to estimate the ability of the SII and other factors to predict poor outcomes. The optimal cutoff point was calculated using the Youden's Index. Boxplots were performed to describe the distribution of admission and day-1 SII in ordinal mRS. Multivariate logistic regression analysis was used to analyze the association between factors and prognosis. Variables included in the model were selected based on the result of univariate analysis. Two-tailed $p<0.05$ was considered significant.

\section{RESULTS}

We enrolled 291 patients (Supplementary Figure 1). Of these, $98(34 \%)$ achieved favorable outcomes at 90 days. The poor outcome group included more females ( 38 vs. $23 \%, p=0.02$ ), older patients ( $59 \pm 14$ vs. $55 \pm 13, p=0.0002)$, lower Glasgow Coma Scales [8 (6-13) vs. 13 (13-15), $p<0.0001$ ], larger ICH volumes [32 (14-59) vs. $12(4-24), p<0.0001]$, and more intraventricular hematomas (64 vs. $27 \%, p<0.001$ ) (Table 1). Admission ANC and day-1 ANC were significantly higher in patients with unfavorable outcome, and they tended to have a lower ALC on day-1. Admission SII and day-1 SII both showed significant differences between outcome groups (Table 1 and Supplementary Table 1). The interval of the day- 1 test in the full cohort was 29 (13-51) h from the onset; no difference was found between both groups.

The multivariate analysis was carried out considering factors including SII, sex, age, ICH volume (logarithm), IVH extension, hematoma location, and craniotomy. In multivariate analysis, day-1 SII independently predicted 90 -day poor outcome (OR $1.74,95 \% \mathrm{CI}=1.03-3.00, p=0.04)$, while admission SII did not (OR 1.19, 95\% 0.81-1.75, $p=0.37$ ) (Table 2). Receiver operating characteristics yielded a cutoff of $1,315 \times 10^{9} / \mathrm{L}$ for admission SII (AUC 0.726 , sensitivity $83.7 \%$, specificity $56.9 \%$ ) and $1,700 \times 10^{9} / \mathrm{L}$ for day-1 SII (AUC 0.699 , sensitivity $53.3 \%$, specificity $77.3 \%$ ) with corresponding maximum Youden index for predicting 90-day outcome (Supplementary Figure 2). Multivariate analysis revealed that Day1-SII $>1,700 \times 10^{9} / \mathrm{L}$ (OR 2.36, 95\% CI $=1.09-5.26, p=0.03$ ), but not admission SII $>1,315 \times 10^{9} / \mathrm{L}(\mathrm{OR} 1.42,95 \% \mathrm{CI}=0.72-2.82, p=0.31)$, was significantly associated with poor 90-day functional outcome (Supplementary Table 2). Moreover, age, GCS, ICH volume, and location were also found as an independent predictor [detailed in(Supplementary Table 2)].

We performed a fitting curve based on the binary functional outcome and their individual ANC, ALC, PLT, and SII to display the trends (Figure 1). A peak of SII occurred at $24-48 \mathrm{~h}$ after 
TABLE 1 | Baseline comparison between 90-day outcome groups.

\begin{tabular}{|c|c|c|c|c|}
\hline & $\begin{array}{l}\text { Full cohort } \\
(n=291)\end{array}$ & $\begin{array}{l}\text { Favorable outcome } \\
\qquad(n=98)\end{array}$ & $\begin{array}{l}\text { Poor outcome } \\
\quad(n=193)\end{array}$ & $P$-value \\
\hline Age, years & $57(14)$ & $55(13)$ & $59(14)$ & $0.0002^{*}$ \\
\hline Onset to admission time, $\mathrm{h}$ & $5(3-8)$ & $6(3-10)$ & $5(3-8)$ & $0.20^{\ddagger}$ \\
\hline Admission SBP, mmHg & $166(143-183)$ & $163(144-181)$ & $166(142-184)$ & $0.71^{\ddagger}$ \\
\hline Admission SII, $\times 10^{9} / \mathrm{L}$ & $1298(658-2244)$ & $989(570-1867)$ & 1440 (792-2422) & $0.004^{\ddagger}$ \\
\hline Day $-1 \mathrm{SII}, \times 10^{9} / \mathrm{L}$ & $1467(884-2485)$ & 969 (685-1564) & $1833(1170-2955)$ & $<0.0001^{\ddagger}$ \\
\hline Craniotomy & $63(22 \%)$ & $12(12 \%)$ & $51(26 \%)$ & $0.008^{\dagger}$ \\
\hline $\mathrm{ICH}$ volume, $\mathrm{ml}$ & $24(9-47)$ & $12(4-24)$ & $32(14-59)$ & $<0.0001^{\ddagger}$ \\
\hline Intraventricular hematoma & $145(50 \%)$ & $22(27 \%)$ & $123(64 \%)$ & $<0.0001^{\dagger}$ \\
\hline
\end{tabular}

Data are mean (standard deviation) or median (interquartile range) for continuous variables, and $n$ (\%) for categorical variables. SBP, systolic blood pressure; DBP, diastolic blood pressure; GCS, glasgow coma scale; SII, systemic immune-inflammation index; ICH, intracerebral hemorrhage.

${ }^{*} x 2$ test or Fisher exact test.

${ }^{\dagger}$ Two-sample Student's t test.

‡ann-Whitney test.

TABLE 2 | Relationship of SII and SII threshold with 90-day predicting poor outcome.

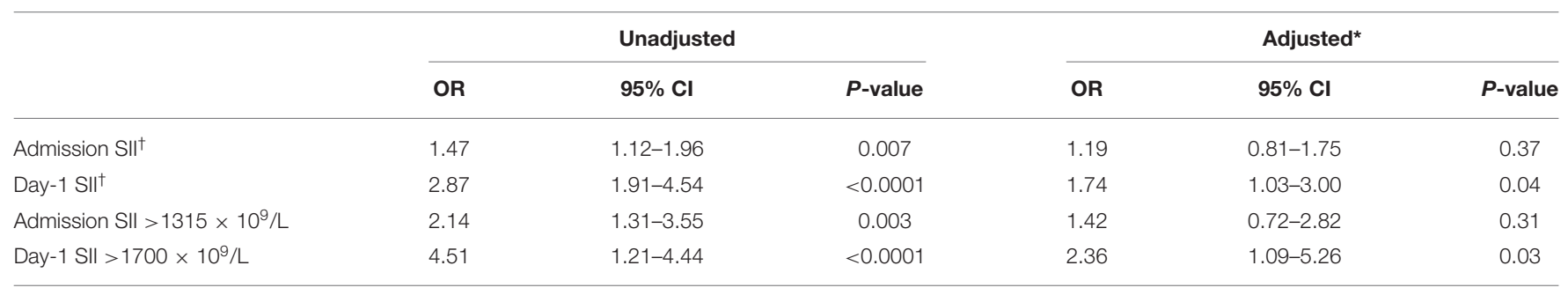

SII, systemic immune-inflammation index; *Adjusted by sex, age, admission Glasgow Coma Scale, logarithm intracerebral hematoma volume, intraventricular hematoma occurrence, hematoma location and craniotomy; ${ }^{\dagger}$ Logarithm.

stroke, and an obvious gap was identified after $24 \mathrm{~h}$ from ICH onset. ANC showed the same trend as SII, and ALC showed an inverse curve. PLT did not show a significant difference on the fitting curve. Furthermore, boxplots of admission and day-1 SII stratified by ordinal mRS revealed different distribution of SII, especially day-1 SII, in each mRS category (Figure 2).

\section{DISCUSSION}

We described the dynamic change of the SII in ICH patients and detected a peak at $24-48 \mathrm{~h}$ after ICH onset. The admission SII and day-1 SII was found to be associated with 90 -day poor outcome. In the multivariate analysis, only day- 1 SII independently predicted 90-day functional outcome with an optimal cutoff at $1,700 \times 10^{9} / \mathrm{L}$. These findings suggest that the SII might serve as a new important indicator for prognosis prediction and risk stratification in ICH patients. To our knowledge, this was the first study that reported the dynamic change of SII following ICH and evaluated the predicting value of SII in long-term functional outcome in ICH patients.
There is accumulating evidence that inflammatory indices calculated based on routine blood count such as neutrophils and lymphocytes can provide valuable prognostic information in various diseases including ICH and ischemic stroke $(9,24-$ 26). Since these inflammatory indices are easily obtainable and widely accessible, they could be added as simple predictive tools for risk stratification during clinical estimation. Meanwhile, the increase of these inflammatory indices might also reflect the acute inflammatory response to the primary and secondary brain injury.

After ICH occurs, plasma-derived factors (i.e., thrombin and vitronectin) and components released following erythrocyte lysis (i.e., hemoglobin, peroxiredoxin 2, and carbonic anhydrase 1) can activate macrophage/microglia and trigger the inflammatory cascade $(27,28)$. Activated macrophages/microglia further release pro-inflammatory cytokines and chemokines and promote infiltration of peripheral inflammatory cells (29). Neutrophils are the earliest white blood cells recruited from peripheral blood to the brain in response to acute inflammatory immune response (10). In animal models, neutrophil infiltration 

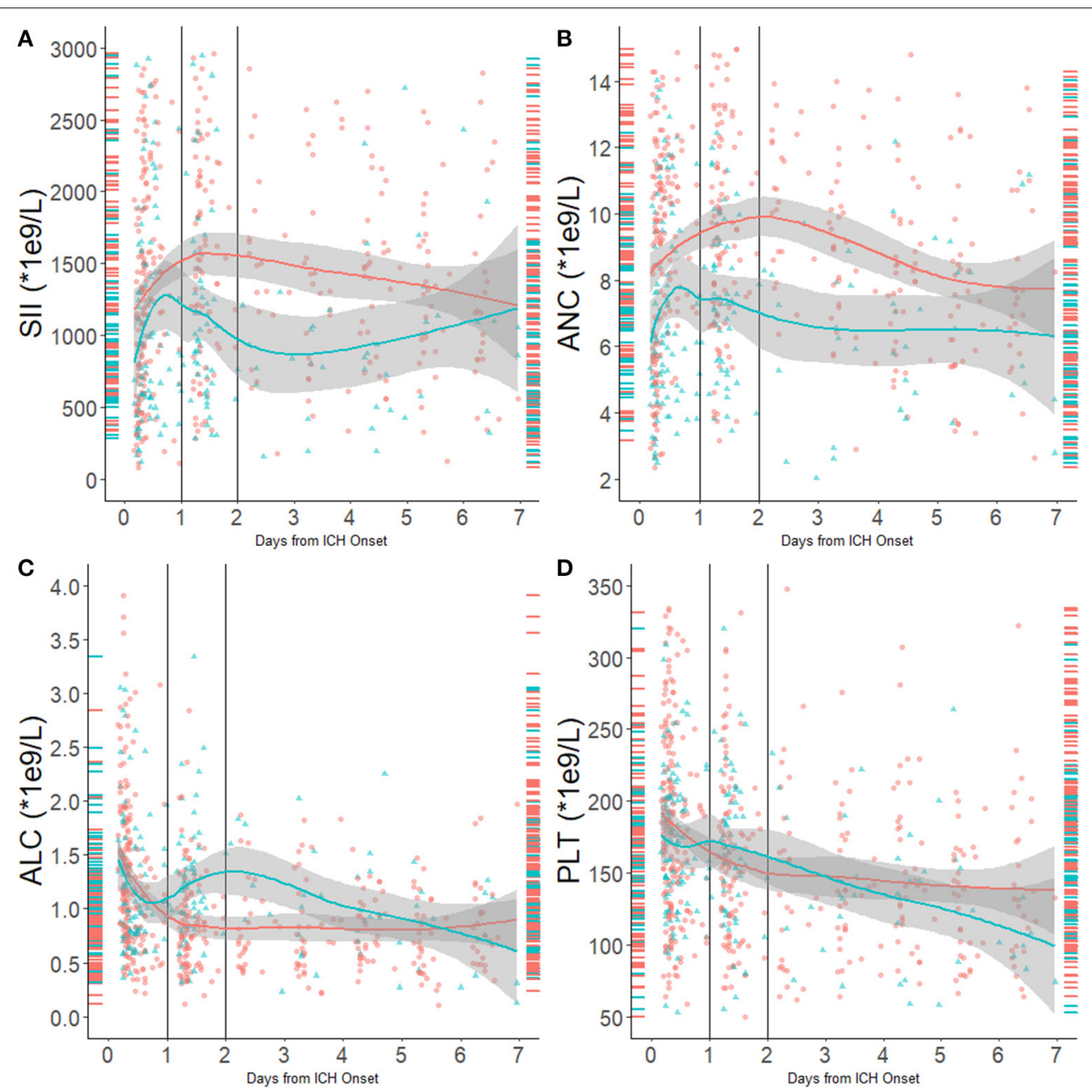

FIGURE 1 | (A) SIl distribution and fitting curve according to 90-day outcome; (B) ANC distribution and fitting curve according to 90-day outcome; (C) ALC distribution and fitting curve according to 90-day outcome; (D) PLT distribution and fitting curve according to 90-day outcome. Red indicates patients with unfavorable outcomes, and blue indicates patients with favorable outcomes. Right-side rungs indicate SII distribution in the first $12 \mathrm{~h}$ from onset; the left side indicates Sll distribution $24-48 \mathrm{~h}$ from onset.

was observed around the hematoma within $4 \mathrm{~h}$ and reached a peak in 1-3 days after $\operatorname{ICH}(6,30)$. By analyzing the tissues surrounding the hematoma in patients with $\mathrm{ICH}$, there was neutrophil infiltration within $8 \mathrm{~h}$ that further increased within 1 day (31). Neutrophils induce neurotoxicity by releasing pro-inflammatory cytokines (i.e., TNF- $\alpha$ and IL-1 $\beta$ ), further contributing to increased capillary permeability, blood-brain barrier destruction, and aggravation of brain edema (32). In preclinical research, targeting neutrophil inhibition alleviated myelin fragmentation and axonal damage, further improving functional outcomes after ICH (33).

Platelets are an integral component of the hemostatic system (28). The balance of platelet aggregation is broken after $\mathrm{ICH}$. The increase of platelet counts in the peripheral circulation induces a hypercoagulable state, which increases the risk of poor outcomes (34). Activated platelets release a series of potent chemical mediators (i.e., adenosine diphosphate, serotonin, thromboxane
A2, and TGF $\beta$ ), all of which may potentially play important roles in brain damage and unfavorable prognosis (28).

In the acute phase after $\mathrm{ICH}$, the sympathetic system and hypothalamic-pituitary-adrenal axis are overactivated and the levels of catecholamines and steroids increase, which contribute to systemic immunosuppression and further induce functional inactivation and apoptosis of peripheral lymphocytes (35). Lymphocytes play a crucial role in immune regulation and host defense against pathogens (10). Decreases in lymphocyte numbers reduces the immune capacity, increases the risk of infection after $\mathrm{ICH}$, and may have an impact on functional outcomes $(34,36)$.

Based on previous studies, the inflammatory immune response may not be reflected in laboratory tests within the first few hours of onset (31). In our cohort, the median onset to admission time was $5 \mathrm{~h}$, which is substantially shorter than that of a previous report (21); as a result, the immune response may not 


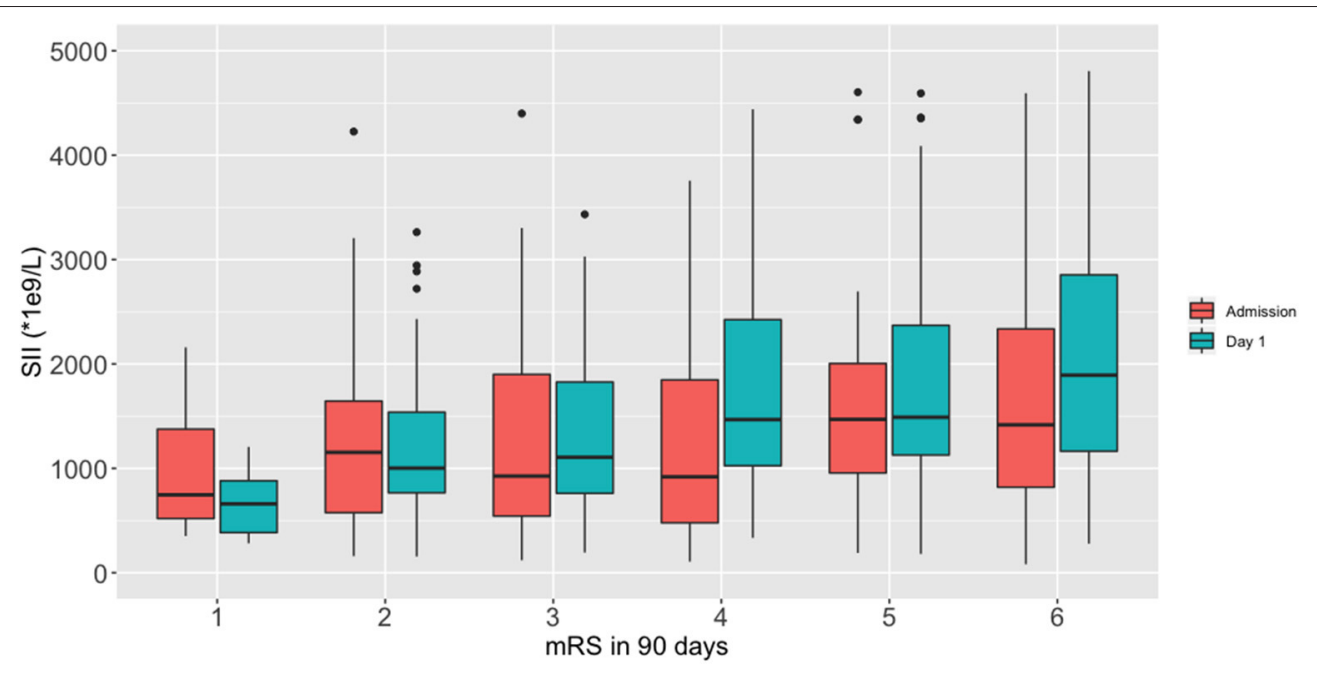

FIGURE 2 | Boxplots of admission and day-1 SIl stratified by ordinal mRS.

fully reach the ultra-acute phase, giving rise to a different result. A more precise observation of inflammatory immune markers in stroke patients may be needed in the future.

There are some limitations in our study. A single-center retrospective cohort has potential biases. Other inflammatory markers such as edema volume and interleukins were not collected, and the interaction with other infective complications were not studied. The strengths of our report include the dynamic change of the immune-inflammation index. A previous study focused on the admission time point but did not consider the actual time from onset of stroke (21). We also had a relatively wide enrollment of patients with $\mathrm{ICH}$, including all locations of hematoma and surgical patients.

\section{CONCLUSION}

SII is an easily calculated index that showed decent ability to predict outcome following ICH. Further investigations may increase the understanding of immune-inflammation processes in $\mathrm{ICH}$ and may guide clinical practice.

\section{DATA AVAILABILITY STATEMENT}

The data and code that support the findings of this study are available from the corresponding author upon reasonable request.

\section{REFERENCES}

1. Johnson CO, Nguyen M, Roth GA, Nichols E, Alam T, Abate D, et al. Global, regional, and national burden of stroke, 1990-2016: a systematic analysis for the Global Burden of Disease Study 2016. Lancet Neurol. (2019) 18:439-58. doi: 10.1016/S1474-4422(19)30034-1

2. Hemphill JC, Bonovich DC, Besmertis L, Manley GT, Johnston SC. The ICH score. Stroke. (2001) 32:891-7. doi: 10.1161/01.str.32.4.891

\section{ETHICS STATEMENT}

The studies involving human participants were reviewed and approved by Medical ethics committee of Sichuan University. Written informed consent for participation was not required for this study in accordance with the national legislation and the institutional requirements.

\section{AUTHOR CONTRIBUTIONS}

YL and XH designed the study. YL, YC, FZ, MY, and HX collected the data. YL and DW analyzed the data. DW, WC, and XH did major revision. All authors contributed to the article and approved the submitted version.

\section{FUNDING}

This work was supported by the National Natural Science Foundation of China (Nos. 81601155 and 81701292).

\section{SUPPLEMENTARY MATERIAL}

The Supplementary Material for this article can be found online at: https://www.frontiersin.org/articles/10.3389/fneur. 2021.628557/full\#supplementary-material

3. Poon MTC, Fonville AF, Salman RAS. Long-term prognosis after intracerebral haemorrhage: systematic review and meta-analysis. J Neurol Neurosurg Psychiatry. (2014) 85:660-7. doi: 10.1136/jnnp-20 13-306476

4. Phan TG, Krishnadas N, Lai VWY, Batt M, Slater LA, Chandra RV, et al. Metaanalysis of accuracy of the spot sign for predicting hematoma growth and clinical outcomes. Stroke. (2019) 50:2030-6. doi: 10.1161/STROKEAHA.118. 024347 
5. Shao Z, Tu S, Shao A. Pathophysiological mechanisms and potential therapeutic targets in intracerebral hemorrhage. Front Pharmacol. (2019) 10:1079. doi: 10.3389/fphar.2019. 01079

6. Zhou Y, Wang Y, Wang J, Anne Stetler R, Yang QW. inflammation in intracerebral hemorrhage: from mechanisms to clinical translation. Prog Neurobiol. (2014) 115:25-44. doi: 10.1016/j.pneurobio.2013.11.003

7. Gusdon AM, Nyquist PA, Torres-Lopez VM, Leasure AC, Falcone GJ, Sheth $\mathrm{KN}$, et al. Perihematomal edema after intracerebral hemorrhage in patients with active malignancy. Stroke. (2020) 51:129-36. doi: 10.1161/STROKEAHA.119.027085

8. Volbers B, Giede-Jeppe A, Gerner ST, Sembill JA, Kuramatsu JB, Lang S, et al. Peak perihemorrhagic edema correlates with functional outcome in intracerebral hemorrhage. Neurology. (2018) 90:e1005-e12. doi: 10.1212/WNL.0000000000005167

9. Lattanzi S, Cagnetti C, Provinciali L, Silvestrini M. Neutrophil-to-lymphocyte ratio predicts the outcome of acute intracerebral hemorrhage. Stroke. (2016) 47:1654-7. doi: 10.1161/STROKEAHA.116.013627

10. Lattanzi S, Brigo F, Trinka E, Cagnetti C, Di Napoli M, Silvestrini M. Neutrophil-to-lymphocyte ratio in acute cerebral hemorrhage: a system review. Transl Stroke Res. (2019) 10:137-45. doi: 10.1007/s12975-018-0649-4

11. Zhang W, Shen Y. Platelet-to-lymphocyte ratio as a new predictive index of neurological outcomes in patients with acute intracranial hemorrhage: a retrospective study. Med Sci Monit. (2018) 24:441320. doi: 10.12659/MSM.910845

12. Qi H, Wang D, Deng X, Pang X. Lymphocyte-to-monocyte ratio is an independent predictor for neurological deterioration and 90-day mortality in spontaneous intracerebral hemorrhage. Med Sci Monit. (2018) 24:928291. doi: 10.12659/MSM.911645

13. Hagen M, Sembill JA, Sprügel MI, Gerner ST, MadŽar D, Lücking H, et al. Systemic inflammatory response syndrome and long-term outcome after intracerebral hemorrhage. Neurol Neuroimmunol Neuroinflammation. (2019) 6:e588. doi: 10.1212/NXI.0000000000000588

14. Hu B, Yang XR, Xu Y, Sun YF, Sun C, Guo W, et al. Systemic immune-inflammation index predicts prognosis of patients after curative resection for hepatocellular carcinoma. Clin Cancer Res. (2014) 20:621222. doi: 10.1158/1078-0432.CCR-14-0442

15. De Giorgi U, Procopio G, Giannarelli D, Sabbatini R, Bearz A, Buti S, et al. Association of systemic inflammation index and body mass index with survival in patients with renal cell cancer treated with nivolumab. Clin Cancer Res. (2019) 25:3839-46. doi: 10.1158/1078-0432.CCR-18-3661

16. Jomrich G, Paireder M, Kristo I, Baierl A, Ilhan-Mutlu A, Preusser M, et al. High systemic immune-inflammation index is an adverse prognostic factor for patients with gastroesophageal adenocarcinoma. Ann Surg. (2019) 273:532-41. doi: 10.1097/SLA.0000000000003370

17. Yang Y-L, Wu C-H, Hsu P-F, Chen S-C, Huang S-S, Chan WL, et al. Systemic immune-inflammation index (SII) predicted clinical outcome in patients with coronary artery disease. Eur J Clin Invest. (2020) 50:e13230. doi: 10.1111/eci.13230

18. Hou D, Wang C, Luo Y, Ye X, Han X, Feng Y, et al. Systemic immuneinflammation index (SII) but not platelet-albumin-bilirubin (PALBI) grade is associated with severity of acute ischemic stroke (AIS). Int J Neurosci. (2020) 1-6. doi: 10.1080/00207454.2020.1784166

19. Wang BL, Tian L, Gao XH, Ma XL, Wu J, Zhang CY, et al. Dynamic change of the systemic immune inflammation index predicts the prognosis of patients with hepatocellular carcinoma after curative resection. Clin Chem Lab Med. (2016) 54:1963-69. doi: 10.1515/cclm-2015-1191

20. Zhou Z, quan, Pang S, Yu X, chen, Xue Q, et al. Predictive values of postoperative and dynamic changes of inflammation indexes in survival of patients with resected colorectal cancer. Curr Med Sci. (2018) 38:798808. doi: 10.1007/s11596-018-1946-6

21. Trifan G, Testai FD. Systemic immune-inflammation (SII) index predicts poor outcome after spontaneous supratentorial intracerebral hemorrhage. J Stroke Cerebrovasc Dis. (2020) 29:105057. doi: 10.1016/j.jstrokecerebrovasdis.2020.105057
22. Hu X, Fang Y, Ye F, Lin S, Li H, You C, et al. Effects of plasma Ddimer levels on early mortality and long-term functional outcome after spontaneous intracerebral hemorrhage. J Clin Neurosci. (2014) 21:136467. doi: 10.1016/j.jocn.2013.11.030

23. Wilson JT, Hareendran A, Grant M, Baird T, Schulz UG, Muir KW, et al. Improving the assessment of outcomes in stroke: use of a structured interview to assign grades on the modified Rankin Scale. Stroke. (2002) 33:22436. doi: 10.1161/01.str.0000027437.22450.bd

24. Lattanzi S, Cagnetti C, Provinciali L, Silvestrini M. Neutrophil-to-lymphocyte ratio and neurological deterioration following acute cerebral hemorrhage. Oncotarget. (2017) 8:57489-94. doi: 10.18632/oncotarget.15423

25. Altintas O, Altintas MO, Tasal A, Kucukdagli OT, Asil T. The relationship of platelet-to-lymphocyte ratio with clinical outcome and final infarct core in acute ischemic stroke patients who have undergone endovascular therapy. Neurol Res. (2016) 38:759-65. doi: 10.1080/01616412.2016. 1215030

26. Switonska M, Piekus-Slomka N, Slomka A, Sokal P, Zekanowska E, Lattanzi S. Neutrophil-to-lymphocyte ratio and symptomatic hemorrhagic transformation in ischemic stroke patients undergoing revascularization. Brain Sci. (2020) 10:771. doi: 10.3390/brainsci10110771

27. Keep RF, Hua Y, Xi G. Intracerebral haemorrhage: Mechanisms of injury and therapeutic targets. Lancet Neurol. (2012) 11:72031. doi: $10.1016 /$ S1474-4422(12)70104-7

28. Garton T, Keep RF, Wilkinson DA, Strahle JM, Hua Y, Garton HJL, et al. Intraventricular hemorrhage: the role of blood components in secondary injury and hydrocephalus. Transl Stroke Res. (2016) 7:44751. doi: 10.1007/s12975-016-0480-8

29. Wilkinson DA, Pandey AS, Thompson BG, Keep RF, Hua Y, Xi G. Injury mechanisms in acute intracerebral hemorrhage. Neuropharmacology. (2018) 134(Pt B):240-8. doi: 10.1016/j.neuropharm.2017.09.033

30. Wang $J$, Doré S. Inflammation after intracerebral hemorrhage. $J$ Cereb Blood Flow Metab. (2007) 27:894-908. doi: 10.1038/sj.jcbfm. 9600403

31. Mackenzie JM, Clayton JA. Early cellular events in the penumbra of human spontaneous intracerebral hemorrhage. J Stroke Cerebrovasc Dis. (1999) 8:18. doi: 10.1016/S1052-3057(99)80032-9

32. Chen S, Yang Q, Chen G, Zhang JH. An update on inflammation in the acute phase of intracerebral hemorrhage. Transl Stroke Res. (2014) 6:48. doi: 10.1007/s12975-014-0384-4

33. Sansing LH, Harris TH, Kasner SE, Hunter CA, Kariko K. Neutrophil depletion diminishes monocyte infiltration and improves functional outcome after experimental intracerebral hemorrhage. Acta Neurochir Suppl. (2011) 111:173-8. doi: 10.1007/978-3-7091-0693-8_29

34. Tao C, Wang J, Hu X, Ma J, $\mathrm{Li} \mathrm{H}$, You C. Clinical value of neutrophil to lymphocyte and platelet to lymphocyte ratio after aneurysmal subarachnoid hemorrhage. Neurocrit Care. (2017) 26:393-401. doi: 10.1007/s12028-016-0332-0

35. Meisel C, Schwab JM, Prass K, Meisel A, Dirnagl U. Central nervous system injury-induced immune deficiency syndrome. Nat Rev Neurosci. (2005) 6:775-86. doi: $10.1038 /$ nrn1765

36. Dirnagl U, Klehmet J, Braun JS, Harms H, Meisel C, Ziemssen T, et al. Strokeinduced immunodepression: experimental evidence and clinical relevance. Stroke. (2007) 38(2 Suppl):770-3. doi: 10.1161/01.STR.0000251441.89665.bc

Conflict of Interest: The authors declare that the research was conducted in the absence of any commercial or financial relationships that could be construed as a potential conflict of interest.

Copyright $\odot 2021 \mathrm{Li}$, Wen, Cui, Chen, Zhang, Yuan, Xiao, Li, Ma, Hu and You. This is an open-access article distributed under the terms of the Creative Commons Attribution License (CC BY). The use, distribution or reproduction in other forums is permitted, provided the original author(s) and the copyright owner(s) are credited and that the original publication in this journal is cited, in accordance with accepted academic practice. No use, distribution or reproduction is permitted which does not comply with these terms. 